\title{
Evaluation of Interleukin 6 Levels in Gingival Crevicular Fluid and Periodontal Ligament on Application of Orthodontic Forces
}

\author{
Akhil Shetty ${ }^{1}$, Mahima Jain ${ }^{2}$, K Sneha $^{3}$, Veena Shetty ${ }^{4}$, Shama Rao ${ }^{5}$, Ashutosh Shetty ${ }^{6}$
}

\begin{abstract}
Aim and objective: The purpose of this study was to assess and compare the interleukin 6 levels in gingival crevicular fluid and periodontal ligament before and after application of the orthodontic force.

Materials and methods: It is a split mouth study where the gingival crevicular fluid (GCF) and periodontal ligament (PDL) samples of 20 individuals were collected from control and experimental site. GCF samples were collected using 1-5 $\mu \mathrm{L}$ calibrated volumetric microcapillary pipette. PDL samples were collected from the extracted tooth, and PDL tissue was scraped using Gracey curette and was immediately transferred to Eppendorf microcentrifuge tubes (Sigma-Aldrich; St. Louis, MO, USA). The collected samples were stored at $-80^{\circ} \mathrm{C}$ for further analysis using ELISA kit (Human IL-6 ELISA MAX ${ }^{\mathrm{TM}}$ Delux-5 plates) and the optical density was recorded at $450 \mathrm{~nm}$.

Result: In the study it was observed that there was an increase in IL- 6 concentration in the PDL in the experimental group (3rd day of force application). The concentration of IL- 6 was recorded after 3rd day of force application between the experimental groups of PDL and GCF and an increase in the levels of IL- 6 was noted. The increase in the level of IL-6 in these groups was statistically insignificant.

Conclusion: The study depicted similar concentration level of IL-6 in GCF and PDL on the 3rd day of application of orthodontic force. Therefore, GCF can be used as a biomarker in the assessment of IL-6 for up to 3 days of force application instead of PDL.

Clinical significance: GCF can be assessed to know the concentration of cytokines (IL-6) upto 3 days of force application in the PDL, as collection of GCF is a non-invasive technique.

Keywords: ELISA, Gingival crevicular fluid, Interleukin 6, Orthodontic force, Periodontal ligament.

World Journal of Dentistry (2022): 10.5005/jp-journals-10015-1891
\end{abstract}

\section{INTRODUCTION}

Tooth movement is influenced by the application of force on periodontal ligament (PDL) and remodeling of alveolar bone. In PDL tissue, the inflammatory process is initiated when a mechanical stimulus is exerted on a tooth. Biological processes of tooth movement associated with alveolar bone resorption and apposition is triggered by inflammatory mediators.

Mechanical forces such as orthodontic force or masticatory forces, transmits the stress from tooth to the alveolar bone through the PDL. The PDL is abundant with fibrillar collagen and extracellular matrix which is metabolically active and helps in translating the applied force to the periodontium. The PDL integrity is maintained by the PDL cells which act as stem cells and help in regeneration and remodeling of periodontal tissue during application of orthodontic force. ${ }^{2}$ Inflammatory mediators responsible for remodeling of connective tissue and bone are secreted by PDL cells.

Upon application of mechanical stress there are various molecular changes that are induced in the PDL and GCF. GCF is a transudate that appears from the interstitial tissues and is an amalgamation of plaque, cytokines, blood molecules, enzymes, biofilm, and host tissue. In conditions where bone formation/remodeling occurs, such as periodontitis and orthodontic tooth movement, GCF and the PDL release different types of mediators.

It can therefore be said that the periodontal health of an individual can be assessed by the GCF. ${ }^{3}$ Despite the evidence, it suggests that the change in the PDL directly affects GCF, there
${ }^{1-3,6} \mathrm{AB}$ Shetty Memorial Institute of Dental Sciences, NITTE (Deemed to be University), Mangaluru, Karnataka, India

${ }^{4}$ KS Hegde Medical Academy (KSHEMA), NITTE (Deemed to be University), Mangaluru, Karnataka, India

${ }^{5}$ NITTE (Deemed to be University for Stem Cell Research and Regenerative Medicine), Mangaluru, Karnataka, India

Corresponding Author: Akhil Shetty, Department of Orthodontics and Dentofacial Orthopaedics, AB Shetty Memorial Institute of Dental Sciences, NITTE (Deemed to be University), Mangaluru, Karnataka, India, Phone: +919986785660, e-mail: drakhilshetty@nitte.edu.in

How to cite this article: Shetty A, Jain M, Sneha K, et al. Evaluation of Interleukin 6 Levels in Gingival Crevicular Fluid and Periodontal Ligament on Application of Orthodontic Forces. World J Dent 2022;13(1):16-20.

Source of support: Nil

Conflict of interest: None

are limited studies which have evaluated the expression of interleukin- 6 in PDL and GCF.

Cytokines are considered as key mediators involved in bone remodeling under physiologic and mechanical loading-induced conditions. Experiments have shown that interleukin-1 beta is one of the earliest bone resorption markers. Prostaglandin E2, interleukin- 6 and related cytokines also increases during the resorption activity of bone. The secretion of cytokines in the PDL depends on the application of orthodontic forces. Since there is a

(c) The Author(s). 2022 Open Access This article is distributed under the terms of the Creative Commons Attribution 4.0 International License (https://creativecommons.org/licenses/by-nc/4.0/), which permits unrestricted use, distribution, and non-commercial reproduction in any medium, provided you give appropriate credit to the original author(s) and the source, provide a link to the Creative Commons license, and indicate if changes were made. The Creative Commons Public Domain Dedication waiver (http://creativecommons.org/publicdomain/zero/1.0/) applies to the data made available in this article, unless otherwise stated. 
continuous flow of GCF in the PDL, the cytokine level in GCF is not dependent on the pressure side or the compression side. ${ }^{4}$

IL- 6 regulates the bone remodeling process by interacting with bone cells. Increase in IL- 6 expression is seen in the periodontal tissue on application of orthodontic forces.

The level of cytokines can be used as an indicative marker during orthodontic treatment and help in treatment planning. ${ }^{4}$ Thus, knowledge of the ongoing process can aid in proper mechanical loading and subsequently shorten the treatment duration. Determining the precise force, the duration that should be used can ultimately produce optimal treatment with minimal side effects.

So the aim of this study was to analyze and compare the levels of Interleukin 6 (IL-6) in gingival crevicular fluid and periodontal ligament before and after application of the orthodontic force.

\section{Materials and Methods}

The present study was carried out in the Department of Orthodontics, AB Shetty Memorial Institute of Dental Sciences, Mangaluru. A total of 20 patients between the age-group of 18 and 30 years requiring orthodontic treatment were enrolled in the study. Inclusion Criteria included healthy individuals with good periodontal health, patient who required extraction for the orthodontic treatment, no anti-inflammatory drugs taken

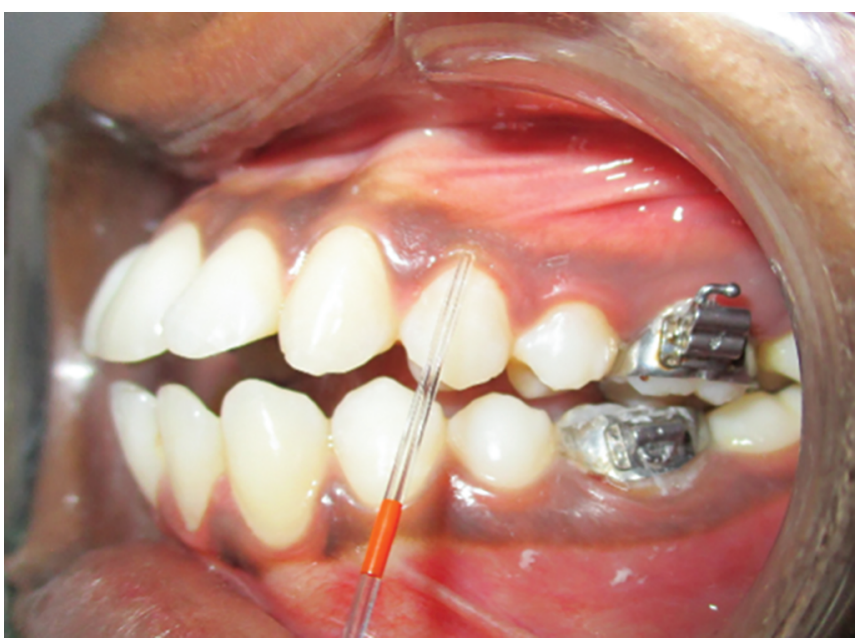

Fig. 1: Collection of GCF sample using micropipette

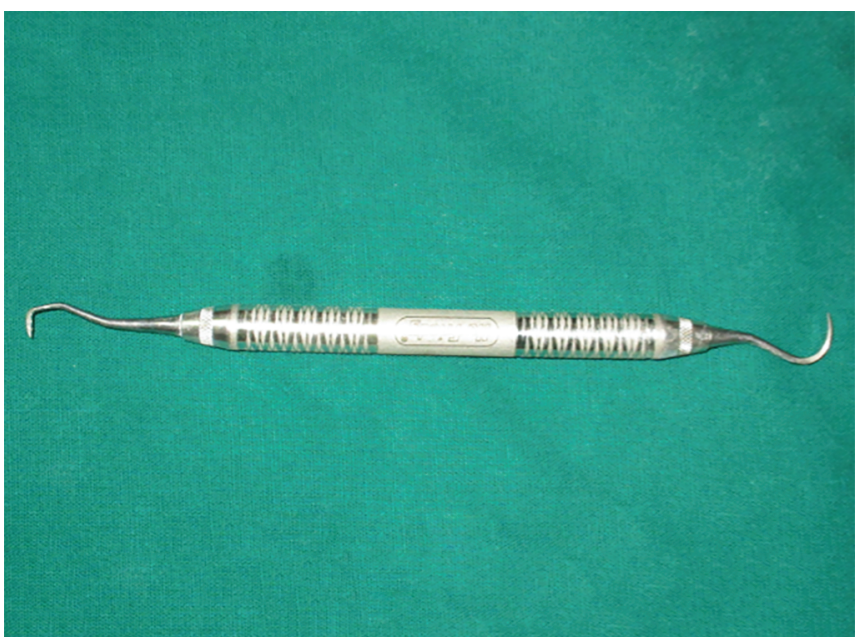

Fig. 2: Gracey curette for scraping the PDL during treatment. Exclusion Criteria included previous history of orthodontic treatment, patient with oral gingival and periodontal infections, patients with poor oral hygiene, pregnant women and pre pubertal patient. Clearance for the study was obtained from the Institutional Ethics Committee and an informed consent was obtained from each patient before the beginning of the study.

It was a split mouth study where the GCF and PDL samples were collected from both the control and the experimental site.

On the control site, GCF samples were collected using 1-5 $\mu \mathrm{L}$ calibrated volumetric micro capillary pipette, from the premolar region just before the extraction (Fig. 1). After the extraction was carried out, PDL was scraped from the extracted tooth using Gracey curette and was immediately transferred to Eppendorf microcentrifuge tubes (Sigma-Aldrich; St. Louis, MO, USA) and preserved (Figs 2 and 3 ).

On the experimental site, brackets $(0.022$ slot MBT mechanotherapy) were bonded (Fig. 4) and an initial intrusive force of 100-150 $\mathrm{g}$ was applied using a cantilever spring on the premolar to be extracted. The force was measured using the correx tension gauge (Haag-Streit, Switzerland). On the third day of force application, GCF samples were collected after which the extraction of the premolar was carried out. Gracey curette was used for scraping the PDL of extracted tooth immediately.

The collected samples were stored at $-80^{\circ} \mathrm{C}$ for further analysis using the ELISA kit (Human IL-6 ELISA MAX ${ }^{\mathrm{TM}}$ Delux-5 plates). The

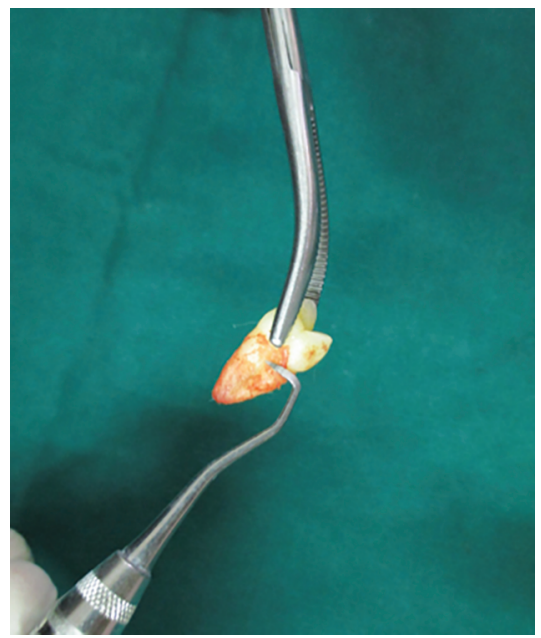

Fig. 3: Procedure for scraping of PDL

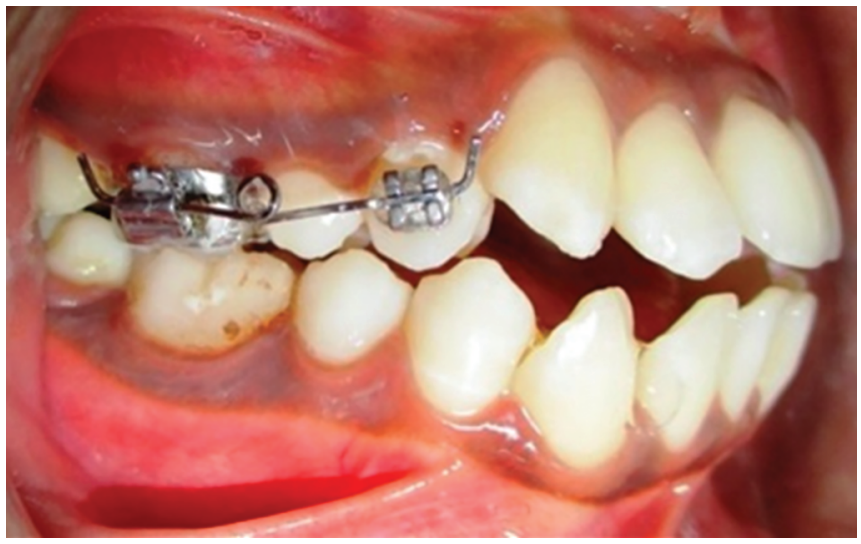

Fig. 4: Cantilever spring for intrusive force using 0.022 slot MBT bracket 
readings were taken from the ELISA reader and the optical density was recorded at $450 \mathrm{~nm}$.

\section{Statistical Analysis}

The collected data was summarized by using frequency, percentage, mean and standard deviation. Unpaired $t$-test was used to find differences between the two groups.

" $P$ " values less than 0.05 were considered as statistically significant and SPSS version 22 was used to analyze the data.

\section{Results}

The study included 20 patients ( 11 females and 9 males) with the females having a mean age of 20.8 years and males with a mean age of 21.3 years.

The mean IL- 6 concentrations in gingival crevicular fluid were $4.5950 \pm 1.6668$ in the control group and $4.3975 \pm 1.5948$ in the experimental group, with a $p$ value of 0.628 . Both the control group (without force application) and the experimental group (on the third day of force application) showed no significant differences in Gingival Crevicular fluid levels (Table 1, Fig. 5).

The mean IL- 6 concentration in the periodontal ligament was $4.3067 \pm 1.4052$ in the control group and $4.3816 \pm 1.3831$ in the experimental group, with a $p$ value of 0.757 . There was no discernible difference between the control and experimental groups in the concentration of IL6 (Table 2, Fig. 6).

With a $p$ value of 0.229 , the mean IL- 6 concentration in the control group for GCF is $4.5950 \pm 1.6338$ and for PDL is $4.3067 \pm 1.4052$. No significant difference in the concentration of IL- 6 in the GCF and PDL of the control group was noted (Table 3, Fig. 7).

Table 1: Unpaired $t$-test for IL-6 levels in gingival crevicular fluid between control and experimental group

\begin{tabular}{lccccc}
\hline Group & $N$ & Mean & Standard deviation & $t$ & $p$ \\
\hline Control & 20 & 4.5950 & 1.6338 & \multirow{2}{*}{0} & 0.628 \\
Experimental & 20 & 4.3975 & 1.5948 & & \\
\hline
\end{tabular}

$p<0.05$ Statistically significant

$p>0.05$ Not significant

No significant difference in gingival crevicular fluid control group and experimental group $(p=0.628)$

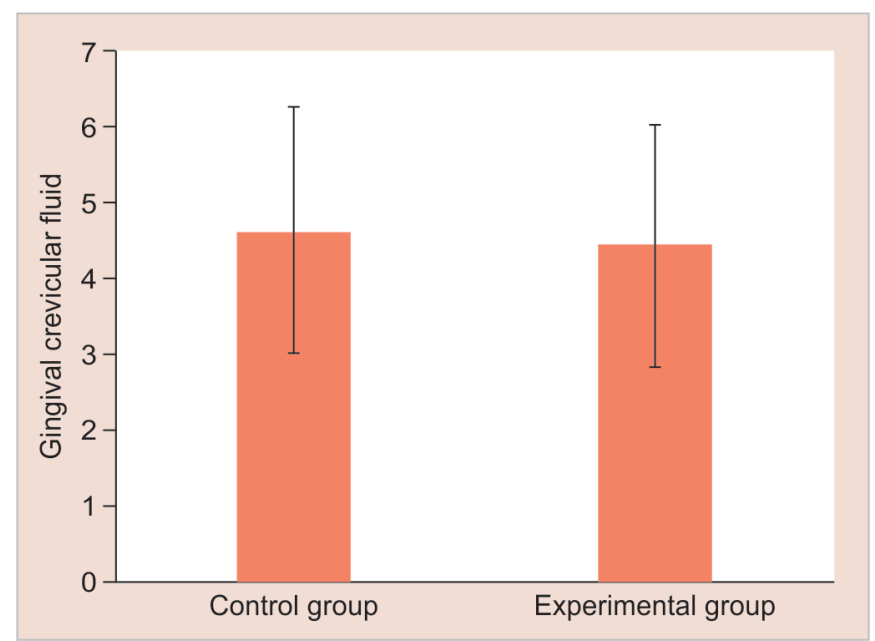

Fig. 5: Overall mean concentration of IL-6 in the gingival crevicular fluid for the experimental and the control group (3rd day of force application)
Table 2: Unpaired $t$-test for IL-6 levels in periodontal ligament between control and experimental group

\begin{tabular}{lccccc}
\hline Group & $N$ & Mean & Standard Deviation & $t$ & $p$ \\
\hline Control & 20 & 4.3067 & 1.4052 & & \\
Experimental & 20 & 4.3816 & 1.3831 & -.314 & 0.757 \\
\hline
\end{tabular}

$p<0.05$ Statistically significant

$p>0.05$ Not significant

No significant difference in periodontal ligament control group and experimental group $(p=0.757)$

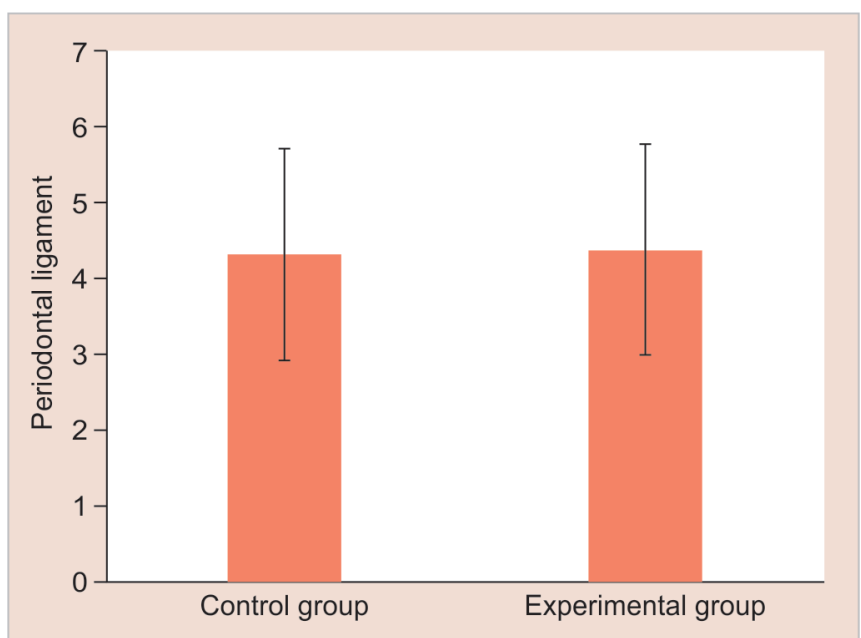

Fig. 6: Overall mean for IL-6 concentration in periodontal ligament for the experimental and the control group (3rd day of force application)

Table 3: Unpaired t-test for IL-6 levels between control groups of gingival crevicular fluid (GCF) and periodontal ligament (PDL)

\begin{tabular}{lccccc}
\hline Group & $N$ & Mean & Standard deviation & $t$ & $p$ \\
\hline GCF control & 20 & 4.5950 & 1.6338 & 1.244 & 0.229 \\
PDL control & 20 & 4.3067 & 1.4052 & &
\end{tabular}

$p<0.05$ Statistically significant

$p>0.05$ Not significant

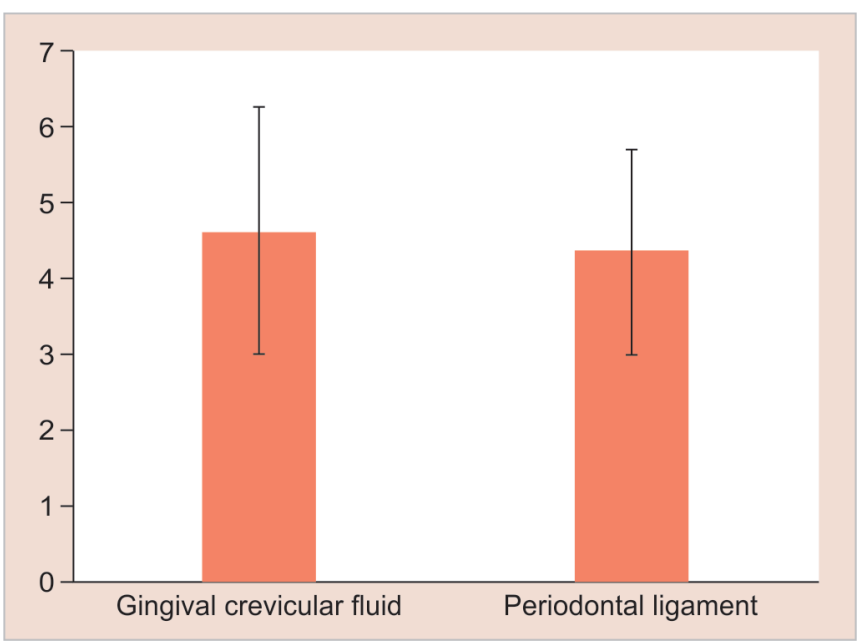

Fig. 7: Overall mean for IL-6 concentration for gingival crevicular fluid and periodontal ligament (without force application) 
Table 4: Unpaired $t$ test for IL-6 levels between test groups of gingival crevicular fluid (GCF) and periodontal ligament (PDL)

\begin{tabular}{lccccc}
\hline Group & $N$ & Mean & Standard Deviation & $t$ & $p$ \\
\hline GCF test & 20 & 4.3975 & 1.5948 & \multirow{2}{*}{046} & 0.963 \\
PDL test & 20 & 4.3816 & 1.3831 & & \\
\hline
\end{tabular}

$p<0.05$ statistically significant

$p>0.05$ Not significant

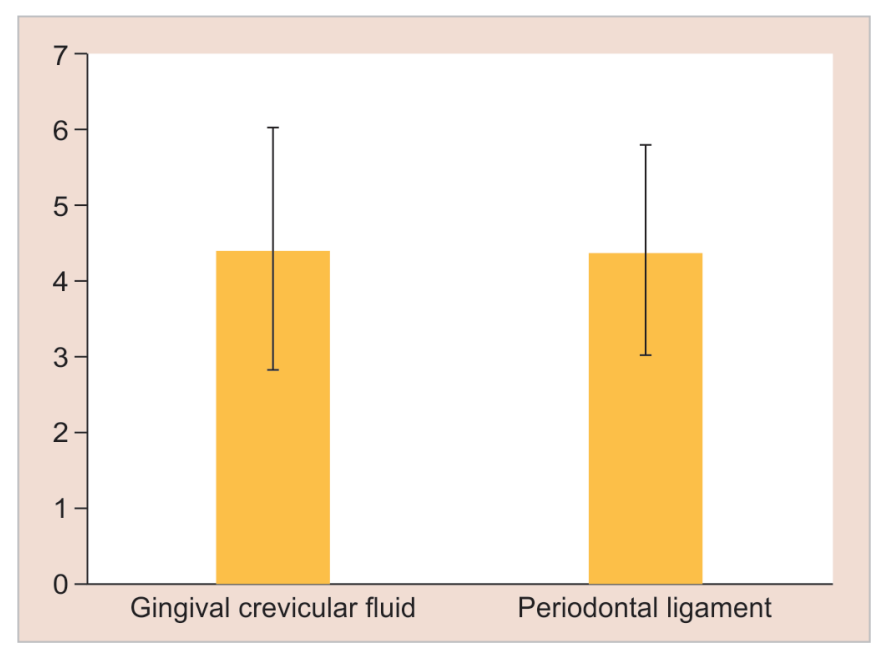

Fig. 8: Overall mean for IL-6 concentration for gingival crevicular fluid and periodontal ligament (3rd day of force application)

GCF had a mean IL- 6 concentration of $4.3975 \pm 1.5948$, while PDL had a mean IL- 6 concentration of $4.3816 \pm 1.3831$, with a $p$ value of 0.963 . No significant difference in the IL- 6 concentration between the GCF and PDL of experimental group was found (Table 4, Fig. 8).

The results depicted no significant difference in the concentration level of IL- 6 in GCF and PDL on the 3rd day of application of orthodontic force.

\section{Discussion}

Application of orthodontic force causes movement of tooth which remodels the paradental tissues, dental pulp, periodontal ligament, gingiva and the alveolar bone. Changes are seen at microscopic levels and macroscopic levels when the tissues are subjected to different magnitude, frequency and amount of force application. ${ }^{5}$ According to numerous studies, a specialized multinucleated giant cell called the osteoclast, which is originated from hematopoietic stem cells facilitates bone resorption. The resorption process is carried out by various mediators which include prostaglandins, cytokines (interleukin-1 beta, interleukin-6 (IL-6) and chemokine (chemokine ligand-2, chemokine ligand-3). These inflammatory mediators alter the cellular activity in the periodontal ligament and trigger the alveolar bone remodeling. ${ }^{6-8}$

This study will be a step towards using the biological principles of tooth movement at the cellular level and utilizing this in improving the clinical approaches and formulating a treatment plan targeted towards these molecules and thus achieving orthodontic tooth movement which is faster with lesser side effects resulting from the orthodontic therapy. Cytokines are involved in initiating, amplifying, perpetuating, and resolving inflammatory responses and are the key mediators in tissue changes and help in tooth movement.
IL-6 also known as B cell stimulatory factor, hepatocyte stimulating factor, B cell growth factor, plasmacytoma growth factor, interferon beta 2 is believed to be working under both the normal physiologic conditions as well as when some external force is applied e.g. during the orthodontic treatment where it tends to upregulate its activity. ${ }^{10,11} \mathrm{IL}-6$ stimulates osteoclast formation, therefore helps in bone resorption. ${ }^{12,14}$

Thus, in the current study, IL- 6 levels are found at baseline concentration in PDL and at the baseline concentrations in GCF and its changing levels in PDL and GCF are compared on the 3rd day of force application.

Various studies have been carried out indicating IL-1 being the primary element in the process of bone resorption, but very limited studies have been conducted to analyze and compare the changing levels of IL- 6 in periodontal ligament and gingival crevicular fluid before and after the application of orthodontic forces. Thus, the present study will help understand the changing levels of IL- 6 concentration in PDL and GCF during the bone remodeling process, thus clarifying its further role in orthodontic tooth movement. In a similar study by Ariffin et al..$^{13}$ the role of interleukin- 6 in stimulation of osteoclasts for bone resorption during orthodontic tooth movement was noted.

Periodontal health can be assessed through GCF secreted in the PDL. Despite the evidence that suggests that the change in the PDL directly affects GCF, there have been limited studies which evaluated the expression of interleukin- 6 in PDL and GCF. ${ }^{3}$ Gingival crevicular fluid analysis is considered as a non-invasive procedure and it gives information about changes in the PDL. Though, it might not be the area specific indicator of periodontal remodeling.

The secretion of cytokines in the PDL depends on the application of orthodontic forces. Since there is a continuous flow of GCF in the PDL, the cytokine level in GCF is not dependent on the pressure or the compression side. ${ }^{4}$ Therefore, considering this fact, the current study aimed at analyzing the IL- 6 levels in the GCF as well as in PDL before and after the application of orthodontic forces.

Madureira et al. ${ }^{4}$ in their study stated that the levels of expression of IL-6, CCL2 and CCL3 vary after the orthodontic force application at different time periods. On day 3 , the results showed that there was an increase in IL- 6 and CCL2 levels. According to the literature, there was a peak increase in the cytokine level on the $3 \mathrm{rd}$ day of application of orthodontic forces. ${ }^{3}$

Alhashimi et al. ${ }^{14}$ in a study on rats demonstrated that messenger RNA expression of IL-1 beta, IL-6, and TNF-alpha reached its highest peak after the force application on 3rd day, and the levels decreased thereafter. Therefore, in this study, day 3 was considered as the experimental time interval, which was two days after the application of orthodontic forces.

As it is stated in literature, the cytokines are expressed in GCF after continuous circulation from PDL and it might take more than 3 days for the IL- 6 to express itself in the GCF. ${ }^{6}$ This is in accordance with the results of the present study which showed no statistically significant increase in the levels of IL- 6 in GCF on the 3rd day of force application.

The results of the present study are not in accordance with the findings of the studies conducted by Madureira et al., ${ }^{3}$ Madureira et al. ${ }^{4}$ and Alhashimi et al. ${ }^{14}$ According to these studies, forces above optimum level leads to tissue necrosis and forces below the optimum range causes no tissue reaction, which prevents the alveolar bone from undergoing frontal resorption. The optimum range of pressure according to the studies is considered as $150-200 \mathrm{~g}$ to achieve a maximum rate of tooth movement at 
the tooth bone interface. ${ }^{9}$ Mohammed et al..$^{15}$ in a study applied $200 \mathrm{~g}$ of force and found a significant increase in the level of IL- 6 at 2 hours, peak on 15 th day. The variation in the present study maybe because of the low magnitude of force used when compared to other studies where they have used a high magnitude of the force of about $200 \mathrm{~g}$.

According to our knowledge, limited studies have been done which simultaneously evaluated and compared the levels of interleukin 6 in GCF and PDL. In this study, the levels of interleukin 6 in GCF and PDL were assessed simultaneously and it showed no significant change in the concentration of IL-6 $(p>0.2)$. The result states that GCF can be assessed to know the concentration of cytokines in the PDL, as collection of GCF is a non-invasive technique.

The entire periodontal ligament was scrapped off from the root surface area of the teeth in the study without considering any specific site, which did not reflect the actual area of the IL- 6 increase, that is, the compression side and the tension sides of the teeth were not differentiated. This could be considered as the limitation of the study. Also, the data was not compared amongst the various age-groups and gender of the subjects. Therefore, the role of IL-6 could not be differentiated according to these aspects and further research might be needed.

\section{Conclusion}

The study depicted a similar concentration level of IL- 6 in GCF and PDL on the 3rd day of application of orthodontic force. Therefore, GCF can be used as a biomarker in the assessment of IL- 6 for up to 3 days of force application instead of PDL as a collection of GCF is a non-invasive technique.

\section{Data Availability Statement}

The data underlying this article cannot be shared publicly for the privacy of individuals that participated in the study. The data will be shared on reasonable request to the corresponding author.

\section{References}

1. Başaran G, Özer T, Kaya FA, et al. Interleukins 2, 6, and 8 levels in human gingival sulcus during orthodontic treatment. Am J Orthod Dentofacial Orthop 2006;130(1):7.e1-7.e6. DOI: 10.1016/j. ajodo.2005.12.027

2. Tantilertanant Y, Niyompanich J, Everts V, et al. Cyclic tensile force-upregulated IL6 increases MMP3 expression by human periodontal ligament cells. Arch Oral Biol 2019;107:104495. DOI: 10.1016/j.archoralbio.2019.104495
3. Madureira DF, da Silva JM, Teixeira AL, et al. Cytokine measurements in gingival crevicular fluid and periodontal ligament: are they correlated? Am J Orthod Dentofacial Orthop 2015;148(2):293-301. DOI: 10.1016/j.ajodo.2015.03.030

4. Madureira DF, de Albuquerque Taddei S, Abreu MH, et al. Kinetics of interleukin- 6 and chemokine ligands 2 and 3 expression of periodontal tissues during orthodontic tooth movement. Am J Orthod Dentofacial Oorthop 2012;142(4):494-500. DOI: 10.1016/j. ajodo.2012.05.012

5. Davidovitch Z, Nicolay OF, Ngan PW, et al. Neurotransmitters, cytokines, and the control of alveolar bone remodeling in orthodontics. Dent Clin North Am 1988;32(3):411-435. DOI: 10.1155/2015/876509

6. Shimizu N, Ogura N, Yamaguchi M, et al. Stimulation by interleukin-1 of interleukin- 6 production by human periodontal ligament cells. Arch Oral Biol 1992;37(9):743-748. DOI: 10.1016/0003-9969(92)90081-i

7. Feller L, Khammissa RA, Schechter I, et al. Biological events in periodontal ligament and alveolar bone associated with application of orthodontic forces. Sci World J 2015;2015. DOI: 10.1155/2015/876509

8. Andrade Jr I, Taddei SR, Souza PE. Inflammation and tooth movement: the role of cytokines, chemokines, and growth factors. Semin Orthod WB Saunders 2012;18(4):257-269.

9. Krishnan V, Davidovitch ZE. Cellular, molecular, and tissue-level reactions to orthodontic force. Am J Orthod Dentofacial Orthop 2006;129(4):469.e1-432.e1. DOI: 10.1016/j.ajodo.2005.10.007

10. Meikle MC. The tissue, cellular, and molecular regulation of orthodontic tooth movement: 100 years after Carl Sandstedt. Eur J Orthod 2006;28(3):221-240. DOI: 10.1093/ejo/cjl001

11. Schröder A, Käppler P, Nazet U, et al. Effects of compressive and tensile strain on macrophages during simulated orthodontic tooth movement. Mediators Inflamm 2020;2020. DOI: 10.1155/2020/2814015

12. Uematsu S, Mogi M, Deguchi T. Interleukin (IL) - 1 $\beta$, IL-6, tumor necrosis factor- $a$, epidermal growth factor, and $\beta 2$-microglobulin levels are elevated in gingival crevicular fluid during human orthodontic tooth movement. J Dental Res 1996;75(1):562-567. DOI: 10.4103/jfmpc.jfmpc_204_19

13. Zainal Ariffin SH, Yamamoto Z, Abidin Z, et al. Cellular and molecular changes in orthodontic tooth movement. Sci World J 2011;11. DOI: 10.1100/2011/761768

14. Alhashimi N, Frithiof $L$, Brudvik P, et al. Orthodontic tooth movement and de novo synthesis of proinflammatory cytokines. Am J Orthod Dentofacial Orthop 2001;119(3):307-312. DOI: 10.1067/mod.2001.110809

15. Mohammed A, Saidath K, Mohindroo A, et al. Assessment and measurement of interleukin 6 in periodontal ligament tissues during orthodontic tooth movement. World J Dent 2019;10(2):88-92. DOi: $10.5005 /$ jp-journals-10015-1610 